\title{
The effects of static versus dynamic stretching on average power in the young-adult athletic population
}

\begin{abstract}
Study design: Single-blind randomized study

Objectives: The purpose of the current study was to compare the effects of static and dynamic stretching in the young-adult athletic population in terms of average knee extensor and flexor muscle power.
\end{abstract}

Background: Stretching prior to exercise is a common practice for many athletic persons, in which varying protocols frequently including static or dynamic stretches. Literature has shown deficits in muscle performance lasting up to one hour as a result of performing static stretching directly prior to activity; alternatively, dynamic stretching has been found to induce positive effects.

Method: Forty young-adult athletes were systematically assigned to static or dynamic stretching groups and performed their respective quadriceps and hamstrings stretches according to their group assignment protocols. Pre-test and post-test measurements of hamstrings and quadriceps average power (Watts) at $60 \% \mathrm{sec}$ and $180 \% \mathrm{sec}$ were obtained using a Biodex Isokinetic Dynamometer.

Results: A mixed design ANOVA showed that there was a significant main effect for stretching type and time (pre-stretch, post-stretch) for dynamic stretching in knee flexor and extensor average power at $60 \%$ sec $(\mathrm{p}<0.05)$, which demonstrates an improvement in quadriceps and hamstrings muscle strength. No significant main effect for stretching type and time was found in knee flexor and extensor average power at $180^{\circ} / \sec (\mathrm{p}>0.05)$.

Conclusion: Dynamic stretching exercises in the form of straight leg kicks and butt kicks may increase quadriceps and hamstring muscle performance at "strength speed" in terms of average power and may be beneficial to young-adult athletes when performed prior to activity.

Contribution of paper

i. Stretching is an important component of warm-up routines for young-adult athletic subjects.

ii. Dynamic stretching increases knee extensor and flexor muscle powers at "strength speeds."

iii. Static stretching does not affect knee extensor and flexor muscle power.

Keywords: dynamic and static stretching exercise, strength, muscle performance
Volume I Issue I - 2016

\author{
Danielle T Freund,' Allison M Liccardo,' \\ Alanna M Rooney,' Ayse Ozcan Edeer,' \\ Valerie Dong Olson² \\ 'Dominican College, Doctor of Physical Therapy Program, USA \\ ${ }^{2}$ Messiah College, Doctor of Physical Therapy Program, USA
}

\begin{abstract}
Correspondence: Ayse Ozcan Edeer,Assistant Professor, Chair,The Research Committee, Dominican College, Doctor of Physical Therapy Program, 470 Western Highway, Orangeburg, NY 10962, USA, Fax 845-398-4892, Tel 845-848-7615, Email ayse.edeer@dc.edu
\end{abstract}

Received: October 14, 2016 | Published: November 21, 2016

\section{Background}

It is a common understanding that many athletic persons perform a warm-up prior to participation in an exercise or sport activity. Although specific warm-up regimes will vary between individuals, it is well known that a traditional approach will include static stretching for the purpose of achieving improved muscular flexibility and joint range of motion. ${ }^{1-3}$ In fact, flexibility training prior to activity has been proven to decrease musculotendinous injuries, ${ }^{4}$ thus supporting the desired goal of obtaining increased flexibility and joint range of motion prior to exercising or participating in a sport. ${ }^{5}$ However, the validity of such claims and the importance of flexibility training prior to activity is not quite certain, as additional reports claim that static stretching prior to activity does not reduce the risk of injury or delayed onset muscle soreness (DOMS), ${ }^{2,6}$ with some studies even suggesting that the risk for injuries directly increases with flexibility. ${ }^{7}$ Additionally, some studies suggest that greater muscle stiffness may be beneficial in the storage and reuse of elastic energy. ${ }^{8}$

Static stretching has been scrutinized in the last decade, with many studies reporting that the practice may elicit negative effects on muscle performance when performed prior to activity. Marek $\mathrm{SM}^{1}$ reported that static stretching was "highly efficient" in reducing muscle performance in terms of strength, specifically for persons between 20 and 30 years of age. ${ }^{1}$ Furthermore, recent studies have reported an additional myriad of muscle performance deficits following sustained static stretching, including maximal voluntary contraction, isometric force, is kinetic torque, one-repetition maximum exercises, vertical jump heights, sprint times, running economy, agility, and balance. . $, 5,7^{-}$ Finally, a study published in 2000 by Fowles et al. ${ }^{9}$ reported deficits in muscle force production lasting up to one hour immediately following 
intense static stretching, warranting further research into the merit and mechanisms behind such claims. ${ }^{9}$

The exact mechanism by which static stretching induces the aforementioned deficits remains unknown; however, it is well known that all aspects of muscle performance are critical to athletic performers and regular exercisers alike. Amiri-Khorasani et al. ${ }^{10}$ suggest that static stretching may decrease force production due to an alteration in the length-tension relationship of the muscle, followed by an altered reflex sensitivity, thereby reducing muscle activation. ${ }^{10}$ Finally, a 2007 study performed by Rubini et al suggested that static stretching may cause distension of the muscle, resulting in the aforementioned deficits. ${ }^{6}$ In contrast, some studies have suggested that dynamic stretching may result in beneficial or null effects when performed prior to activity. ${ }^{3,11,12}$ It is proposed that dynamic stretching prepares the body for physical activity by increasing joint range of motion, as well as blood and oxygen flow to soft tissues, thereby preventing the risk of injury during the subsequent activity. ${ }^{4}$ In fact, McMillian et al. ${ }^{12}$ reported that the United States Army Physical Fitness School (APFS) implemented a dynamic stretching protocol for all military personnel with the same aforementioned goals in addition to increasing body temperature, heart rate, the pliability of joints and muscles, and reaction time. ${ }^{12}$

A study published in 2013 which sought to determine what types of stretching would improve the variations between jumping outcomes on a day-to-day interval concluded that dynamic stretching positively affected vertical jump height and counter-movement jump performance when performed in the morning; the researchers believed that this outcome was a result of dynamic stretching producing a greater increase in short-term maximal performance in the morning when compared to the evening by increasing the core body temperature a greater amount. ${ }^{13}$ Furthermore, a 2013 report by Amiri-Khorasani et al. ${ }^{10}$ suggested that dynamic stretching performed prior to activity may induce greater force production due to post-activation potentiation (PAP) and a higher muscle temperature. PAP is related to an increased peak isometric force and the rate of force production; ${ }^{10}$ this finding is consistent with a 2014 report which found improved movement times in high school female athletes after dynamic stretching when compared to static stretching. ${ }^{14}$ In a conflicting report, Wallmann et al. ${ }^{15}$ compared several types of stretching and their effects on sprint times and concluded that dynamic stretching, along with other forms of stretching, had no beneficial effect on the enhancement of sprint times. ${ }^{15}$ There is much controversy in the literature regarding the effects of static and dynamic stretching. Therefore, the purpose of the current study was to compare the effects of static and dynamic stretching on the average muscle power of the quadriceps and hamstrings muscle groups in young-adult athletes to maximize performance and reduce the risk of injury.

\section{Methods}

The current study focuses on the effects of static and dynamic stretching on average power in the hamstrings and quadriceps muscle groups of young male and female athletic people. The effects of static and dynamic stretching on muscle performance in terms of average power were measured using the Biodex Dynamometer Multi-Joint System (Biodex). The Biodex is a motorized dynamometer that can be adjusted to measure many aspects of muscle performance at various joints of the body. The Biodex has been determined both valid and reliable with an ICC of 0.99 and $0.99-1.00$, respectively, for trial- to-trial testing in terms of position, torque, and velocity. ${ }^{16}$ Average muscular power represents how quickly a muscle can produce force and it is an important measure to individuals who participate in competitive sporting events, but also with those who exercise privately or for leisure. This measure was chosen for the current study to ensure that the conclusions drawn were most relative and meaningful to the target population.

\section{Subjects}

The researchers obtained approval from The Dominican College Institutional Review Board prior to the commencement of the study (IRB \# 2014-0330-05). All potential participants were recruited using convenience and snowball sampling methods. Candidates were required to sign an informed consent form, as well as complete a medical history form to determine eligibility. Inclusion criteria included males and females between the ages of 16 and 25 years who participated in at least 30 minutes of physical activity three times per week. Participants were excluded from the study if their medical history included any current injury or past surgical intervention in one or more lower extremities and if they were currently under the care of a medical doctor for any conditions causing major health concerns. Forty participants ( 20 men, 20 women) satisfied the selection criteria and completed the study. Subject characteristics included a mean age of 22.33 years $( \pm 5.67)$, a mean height of 68.88 inches $( \pm 10.12)$, and a mean weight of 155.66 pounds $( \pm 39.91)$. When surveyed, the most common reported activities that subjects participated in included, in descending order, running, cycling, basketball, weight lifting, and football.

\section{Procedures}

All aspects of the study were performed at a privately owned outpatient orthopedic physical therapy clinic. The participants were blinded to the specific stretch group to which they were assigned. Work distribution within the research team was as follows: Researcher \#1 performed all aspects of managing the participants' paperwork, as well as instructing, monitoring, and time-keeping for each participant's static stretching protocol; Researcher \#2 performed all aspects of testing on the Biodex due to a history of extensive training and knowledge of the machine; Researcher \#3 performed all aspects of instructing, monitoring, and time-keeping for each participant's warm-up and dynamic stretching protocol. Participants arrived at the facility and completed the informed consent and medical history forms. After being deemed appropriate for the study based on the aforementioned inclusion and exclusion criteria, subjects performed a five-minute warm-up on an upright stationary bicycle. Immediately following the warm-up, participants' baseline data was measured using a Biodex Multi-Joint System Dynamometer. The procedure on the Biodex was as follows: Each participant received a brief introduction of the Biodex and instructions to kick (knee flexion and extension) as "fast and hard" as possible to test the quadriceps and hamstrings muscle groups. Prior to each set, the participant completed one trial kick of knee flexion and extension to ensure understanding of the test in avoidance of error. The test was performed at a velocity of $60 \%$ sec (slower speed) for one set of five repetitions on the right lower extremity. Averages of all five repetitions were used for statistical analysis. Subsequently, after a 30 second break, the test was repeated at a velocity of $180^{\circ} / \mathrm{sec}$ (faster speed) for one set of five repetitions. After a quick adjustment to the machine, the testing procedure was repeated on the left lower extremity. All data was recorded and immediately printed from the Biodex after each use, paired with the 
appropriate paperwork, and stored via a number system (\#1-40) to anonymize and protect each participant's confidentiality. When not in direct use, all data was filed and locked in the office. The data for each participant's dominant leg was used for statistical analyses (Table 1).

Table I Test and assessment protocol

\section{Informed consent and medical history \\ 2. Inclusion and exclusion criteria \\ 3. Five-minute warm-up on an upright stationary bicycle \\ 4. Baseline data collection using the Biodex}

\section{- One trial kick of knee flexion and extension \\ - Tests at a velocity of $60^{\circ} / \mathrm{sec}$ for a set of five repetitions on the right lower extremity \\ - $\quad 30$ second break, \\ - Tests at a velocity of $180^{\circ} / \mathrm{sec}$ for a set of five repetitions on the right lower extremity}

- $\quad$ Same test procedure for the left lower extremity

5. Random assignment to one of two stretching groups

6. Stretching protocols

7. Immediate retest on the Biodex.

Participants were randomly assigned to one of two stretching groups. Forty pieces of paper containing one of two group assignments were folded and placed in a box. Each participant removed one piece of paper from the box to receive a group assignment. The static stretching group consisted of each participant performing four 30 -second hamstring stretches by using a looped strap in a supine position. Upon completion, the participant received a two-minute rest before performing four 30 -second quadriceps stretches by using a looped strap in a prone position (Figure 1). The dynamic stretching group consisted of each participant performing two minutes of walking straight-leg kicks. Upon completion, the participant received a twominute rest before performing two minutes of walking butt-kicks (Figure 2). Upon completion of the respective stretching protocol, all participants were immediately retested on the Biodex (Table 2).

Table 2 Stretching protocols

$1 \quad$ Static

a. Four 30-second hamstring stretching by using a looped strap in a supine position

b. Two-minute rest

c. Four 30-second quadriceps stretching by using a looped strap in a prone position

2 Dynamic
a. Two minutes of walking straight-leg kicks
b. Two minutes rest
c. Two minutes of walking butt-kicks
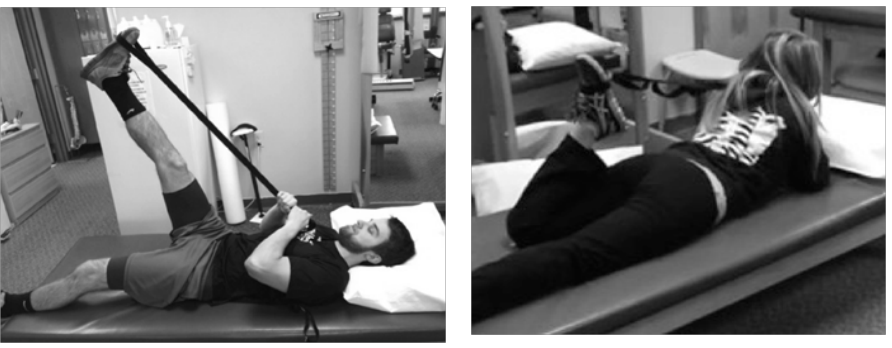

Figure I (A) Participant performing a static stretch to the hamstrings muscle group in a supine position using a looped strap; (B) Participant performing a static stretch to the quadriceps muscle group in a prone position using a looped strap.
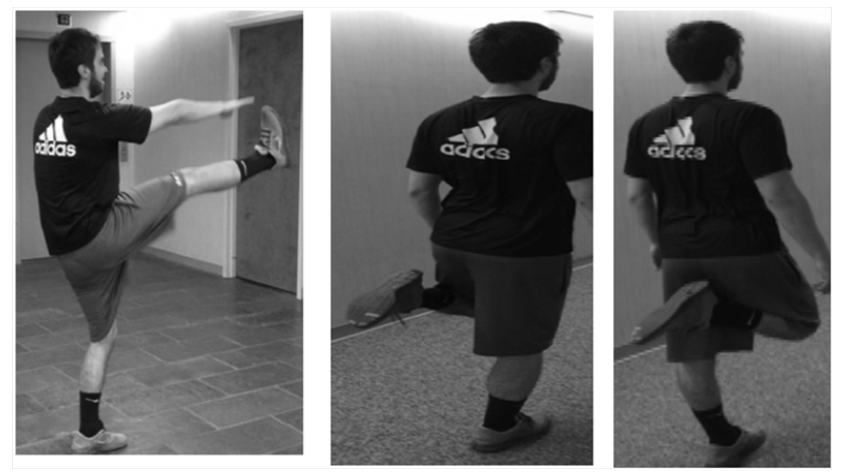

Figure 2 (A) Participant performing a dynamic stretch to the hamstrings muscle group via walking straight-leg kicks; (B) Participant performing a dynamic stretch to the quadriceps muscle group via walking butt-kicks.

\section{Statistical analyses}

All statistical analyses were conducted using IBM Statistical Package for the Social Sciences (SPSS) Version 19.0. An analysis of pre-test to post-test scores for average power obtained via the Biodex was used to determine the effects of both static and dynamic stretching. This pre-test/post-test analysis was performed using a mixed betweenwithin design analysis of variance (mixed design ANOVA). This data was assessed for normal distribution and homogeneity of variance assumptions. Independent t-tests were conducted to further analyze statistical significance between time periods and stretching type. The alpha level for significance was $\mathrm{p}=0.05$ and confidence interval $(\mathrm{CI})$ was set at $95 \%$.

\section{Results}

\section{Knee extensor average power (Watts) at $60 \% \mathrm{sec}$ (Figure 3)}

There was a significant interaction between stretching type and time, Wilks Lambda $=0.820, \mathrm{~F}(1,38)=7.461, \mathrm{p}=0.010$, partial eta squared $=0.164$, showing a large effect size. $T$-test results showed that there was a statistically significant difference for dynamic stretching, $\mathrm{p}=0.000$, suggesting an improvement in average power following a dynamic stretching protocol. There was no statistical significant difference for static stretching, $\mathrm{p}=0.922$. There was a main effect for time showing that dynamic and static stretching approaches affected 
average muscle power, Wilks Lambda $=0.820, \quad \mathrm{~F}(1,38)=8.352$, $\mathrm{p}=0.006$, partial eta squared $=0.180$, showing a large effect size.

\section{Knee flexor average power (Watts) at $60 \% \mathrm{sec}$ (Figure} 4)

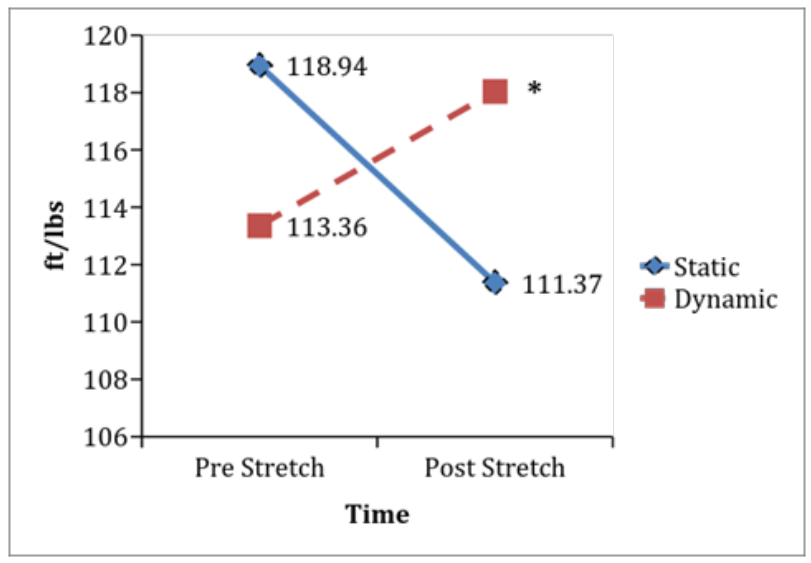

Figure 3 Pre-stretch, post-stretch knee extensor average power (Watts) at $60 \%$ sec between static and dynamic stretching groups (*statistical significant difference).

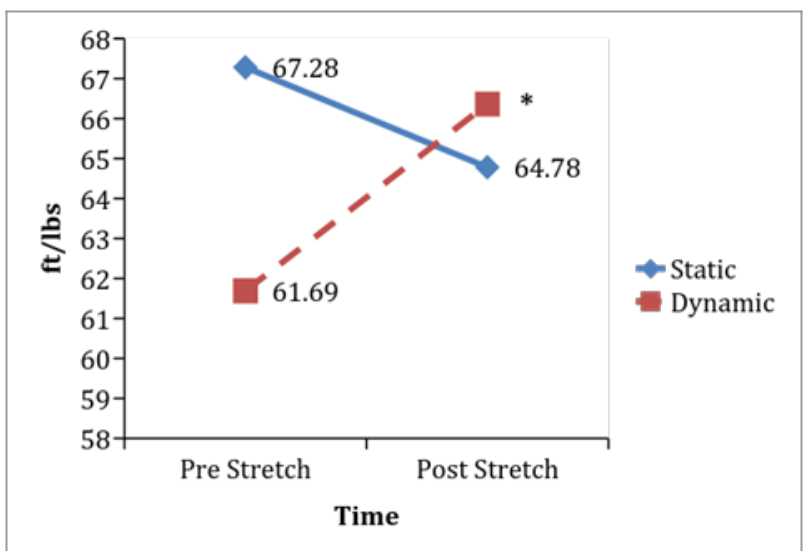

Figure 4 Pre-stretch, post-stretch knee flexor average power (Watts) at $60 \%$ sec between static and dynamic stretching groups (*statistical significant difference).

Between static and dynamic stretching groups across two different time periods (pre-stretch, post-stretch) had a significant interaction between stretching type and time, Wilks Lambda $=0.867$, $\mathrm{F}(1,38)=5.804, \mathrm{p}=0.021$, partial eta squared $=0.133$, showing a large effect size. T-test results showed that there was a statistically significant difference for dynamic stretching, $\mathrm{p}=0.020$, suggesting an improvement in average power following a dynamic stretching protocol. There was no statistically significant difference for static stretching, $p=0.905$. There was a main effect for time showing that dynamic and static stretching approaches affected average muscle power, Wilks Lambda $=0.850, \mathrm{~F}(1,38)=6.687, \mathrm{p}=0.014$, partial eta squared $=0.150$, showing a large effect size.

\section{Knee extensor and flexor average power (Watts) at $180 \%$ sec (Figure 5) (Figure 6)}

Between static and dynamic stretching groups across two different time periods (pre-stretch, post-stretch) had no significant interaction between stretching type and time, $\mathrm{p}>0.05$ for both knee extensor and flexor average power at $60^{\circ} / \mathrm{sec}$. Additionally, there was no main effect for time showing that dynamic and static stretching approaches didn't affect average muscle power, $p>0.05$ for both knee extensor and flexor average power at $60 \% \mathrm{sec}$.

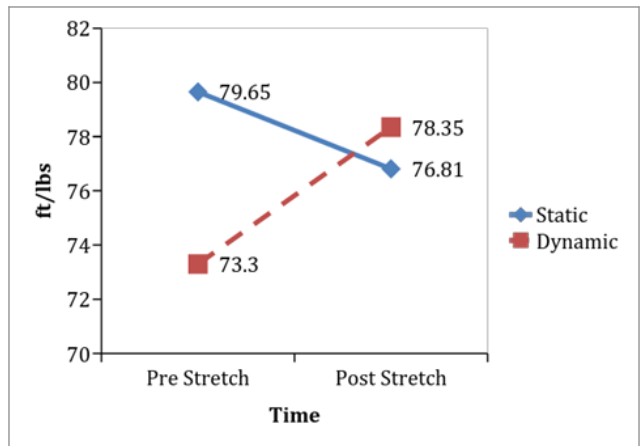

Figure 5 Pre-stretch, post-stretch knee extensor average power (Watts) at $180 \%$ sec between static and dynamic stretching groups.

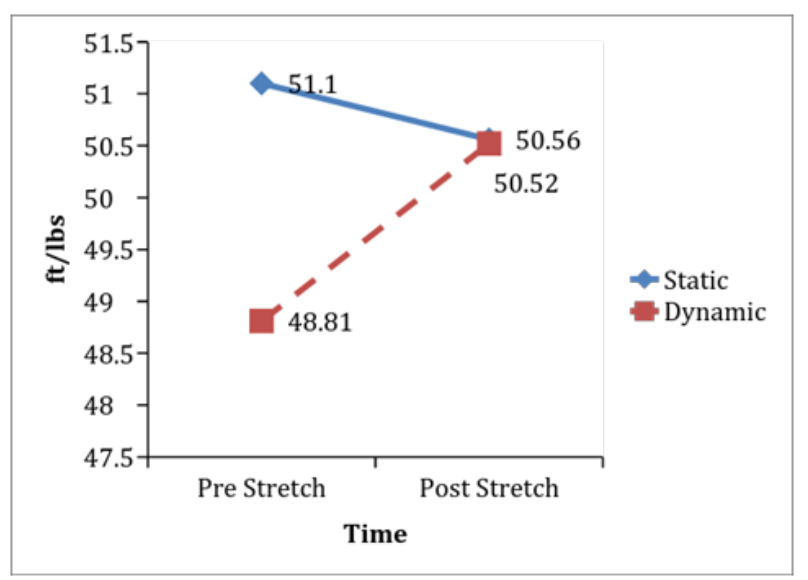

Figure 6 Pre-stretch, post-stretch knee flexor average power (Watts) at $180 \%$ sec between static and dynamic stretching groups.

\section{Between groups comparisons}

The main effect comparing the two types of stretching was not significant, $\mathrm{p}>0.05$ for knee flexor and extensor average power at $60^{\circ} \%$ $\mathrm{sec}$ and $180^{\circ} / \mathrm{sec}$, suggesting no difference in the effectiveness of the two stretching approaches. ${ }^{17-18}$

\section{Discussion}

Stretching, in general, is a staple component of warm-up routines of competitive athletes and regular exercisers alike. Although warmup routines may vary among individuals, static stretching is known to be extremely common. ${ }^{1-3}$ With recent literature raising concerns as to whether static stretching induces performance deficits or not, this study sought-out to determine any truth behind such claims. The researchers chose to test only the hamstrings and quadriceps muscle groups due to the extensive role of these muscles during a majority of sport and leisure physical activities, as well as to compare, support, or deny many similar studies focusing on physical activities primarily using the lower extremities. The researchers chose an age range of 16-25 years in hopes of obtaining a highly conditioned and trained sample to facilitate ease with learning the stretching protocols, to 
attract generally healthy subjects, and to have the potential to make a larger impact with conclusions from the study.

A mixed design ANOVA was conducted to compare pre-test and post-test differences of knee extensor and flexor average power between static and dynamic stretching groups. In terms of dynamic stretching, a statistical significance was found between pre-test and post-test scores at $60 \%$ sec for both knee extension and flexion $(\mathrm{p}=0.000 \& \mathrm{p}=0.020$, respectively). In addition, when compared to pre-test scores, the current study determined a trend of decreasing post-test scores following static stretching, indicating a negative effect in agreement with much of the current literature, ${ }^{1,4,5,7}$ however, no statistical significance was produced. Traditionally, slow speeds have been considered "strength speeds" $(60 \% \mathrm{sec}$ to $120 \% \mathrm{sec})$ and fast speeds $\left(180^{\circ} / \mathrm{sec}\right.$ to $\left.300^{\circ} / \mathrm{sec}\right)$ have been considered "endurance speeds."Based on the results of this study, there are benefits of dynamic stretching in terms of muscular average power when performed at "strength speeds". It is believed that dynamic stretching facilitates an increase in flexibility, blood and oxygen flow to the muscles, and a greater increase in body temperature, thus improving conditions for metabolic reactions during activity to potentially improve muscle performance and decrease the occurrence of injury. ${ }^{4,12}$ The findings of the current study supports several research articles, as well as the research hypothesis, ${ }^{3,4,8,11-14}$ however, further research is warranted to test the effects of dynamic stretching on various muscle groups in the body, as well as to specify the mechanism of the aforementioned enhancements.

Recent literature has reported decreases in many aspects of muscle performance, some lasting up to one hour, following static stretching directly prior to activity, ${ }^{1,4,5,7,9}$ Although results of the current study suggested a negative trend between the pre-test and post-test scores following static stretching, it was unable to produce additional data with statistical significance to support such claims, as well as the research hypothesis. Future research is warranted to examine what factors of the static stretching protocol may be modified to reproduce a negative effect on muscle performance and, if determined, what the mechanism of the effect is. Determining such information will further allow for improved stretching recommendations regarding stretch intensity, duration, and frequency for warm-up routines of all exercisers

Several limitations of the current study exist, including the following: the small sample size $(n=40)$ was obtained via convenience and snowball sampling, as well as a very specific age-range, therefore limiting the ability to generalize the findings of the study to the general population. This study did not include a control group. This study only included the testing of the hamstrings and quadriceps muscle groups, therefore limiting the ability to generalize findings to any muscle in the body. In the static stretching group, participants performed a selfstretch with a looped strap, which is subjective, therefore possibly limiting intensity of the stretch. Finally, multiple subjects were present for testing at any given time which may have created a competitive atmosphere; for example, one individual's post-test scores showed an increase in performance following static stretching, which may have been caused by internal competition or competition among subjects.

\section{Conclusion}

In conclusion, this study determined dynamic stretching in the form of straight-leg kicks and butt-kicks had a positive impact on lower extremity muscle performance at $60^{\circ} / \mathrm{sec}$ (strength speeds) and the potential to be more effective as a warm-up when compared to static stretching. Dynamic stretching proved to be significant for increasing average power in the quadriceps and hamstrings muscle groups of young-adult athletic persons. This increase in average power of the muscle groups may contribute to knee stability and may play an important role in lower extremity injury prevention. This study can help to re-design warm-up routines to include primarily dynamic stretching, which will optimize muscle performance for athletes and regular exercisers of all fitness levels.

\section{Acknowledgements}

None.

\section{Conflict of interest}

The author declares no conflict of interest.

\section{References}

1. Marek SM, Cramer JT, Fincher AL, et al. Acute effects of Static stretching of muscle strength. J Athl Train. 2005;40(2):94-103.

2. Thacker SB, Gilchrist J, Stroup DF, et al. The impact of stretching on sports injury risk: a systematic review of the literature. Med Sci Sports Exerc. 2004;36(3):371-378

3. Weerapong P, Hume P, Kolt G. Stretching: mechanisms and benefits for sport performance and injury prevention. Physical Therapy Reviews. 2004;9(4):189-206.

4. Behm DG, Chaouachi A. A review of the acute effects of static and dynamic stretching on performance. Eur J Appl Physiol. 2011;111(11):2633-2651.

5. Shrier I. Does stretching improve performance? A systematic and critical review of the literature. Clin J Sport Med. 2004;14(5):267-273

6. Rubini EC, Costa AL, Gomes PS. The effects of stretching on strength performance. Sports Med. 2007;37(3):213-224.

7. Bracko MR. Can stretching prior to exercise and sports improve performance and prevent injury? ACSM's Health \& Fitness Journal. 2002;6(5).

8. Sobolewski EJ, Wagner DR, Bressel E. Effect of static stretching and jogging on knee extension isokinetic peak torque. Isokinetics \& Exercise Science. 2011;19(3):157-162.

9. Fowles JR, Sale DG, MacDougall JD. Reduced strength after passive stretch of the human plantarflexors. J Appl Physiol. 2000;89(3):11791188 .

10. Amiri-Khorasani M, Kellis E. Static vs. Dynamic acute stretching effect on quadriceps muscle activity during soccer instep kicking. J Hum Kinet. 2013;39:37-47.

11. Behm DG, Plewe S, Grage P, et al. Relative static stretch- induced impairments and dynamic stretch-induced enhancements are similar in young and middle-aged men. Appl Physiol Nutr Metab. 2011;36(6):790797.

12. McMillian DJ, Moore JH, Hatler BS, et al. Dynamic vs. static-stretching warm up: the effect on power and agility performance. $J$ Strength Cond Res. 2006;20(3):492-499.

13. Chtourou H, Aloui A, Hammouda O, et al. Effect of static and dynamic stretching on the diurnal variations of jump performance in soccer players. PLOS ONE. 2013;8(8):e70534

14. Chatzopoulos D, Galazoulas C, Patikas D, et al. Acute effects of static and dynamic stretching on balance, agility, reaction time and movement time. J Sports Sci Med. 2014;13(2):403-409. 
15. Wallmann HW, Christensen SD, Perry C, et al. The acute effects of various types of stretching static, dynamic, ballistic, and no stretch of the iliopsoas on 40-yard sprint times in recreational runners. Int $J$ Sports Phys Ther. 2012;7(5):540-547.

16. Drouin JM, Valovich-mcLeod TC, Shultz SJ, et al. Reliability and validity of the biodex system 3 Pro Isokinetic Dynamometer velocity, torque and position measurements. Eur J Appl Physiol. 2004;91(1):22-29.
17. Nelson AG, Kokkonen J, Arnall DA. Acute muscle stretching inhibits muscle strength endurance performance. $J$ Strength Cond Res. 2005;19(2):338-343.

18. Trajano GS, Seitz L, Nosaka K, et al. Contribution of central vs. peripheral factors to the force loss induced by passive stretch of the human plantar flexors. J Appl Physiol. 2013;115(2):212-218. 